\title{
An Investigation of Non-Equilibrium Effects in Thermal Argon Plasmas
}

\author{
C. J. Timmermans, R. J. Rosado*, and D. C. Schram \\ Department of Physics, Eindhoven University of Technology, The Netherlands
}

Z. Naturforsch. 40a, 810-825 (1985); received January 30, 1985

The parameters and transport properties of a wall stabilized argon arc $(40-200 \mathrm{~A})$ at atmospheric pressure with diameters of 5 and $8 \mathrm{~mm}$ are studied by spectroscopy and interferometry. The plasma is assumed to be partial local thermal equilibrium and this assumption is verified with the aid of a collisional-radiative model. The departures from Saha-equilibrium of the argon neutral ground state are found to be associated with particle diffusion and the escape of recombination radiation. The measurement of the total excitation rate, from the ground level, including direct ionization, of neutral argon is in reasonable agreement with the literature value.

\section{General Introduction}

The study of the plasma state has always been accompanied by the evaluation of the validity criteria for the description of the plasma state by an equilibrium model. Because of transport and partial radiation escape, laboratory plasmas are not in complete thermodynamic equilibrium and for many applications the local thermal equilibrium (LTE) model is used to determine plasma parameters. The study of the next model in the equilibrium hierarchy, the partial local thermal equilibrium (PLTE) also has gained importance [1-4, 22].

Besides for equilibrium studies, the high current atmospheric pressure arc has also received interest for its applications as plasma spraying, cutting, light sources etc. More recent renewed interest in thermal plasmas for high temperature chemistry has been reported $[5,6]$.

Another line of study is their application as absolute intensity standard [7] both with hydrogen [8] and argon as arc gas especially for the ultra violet spectral region [9-12]. For all applications a good description of the plasma parameters is very important. The validity of the LTE model versus that of the PLTE model becomes of special interest, as the arc plasma is usually close to, but not already in LTE. In the past, several criteria for the validity

\footnotetext{
* At present PULSARR, Eindhoven, The Netherlands. Reprint requests to Dr. C. J. Timmermans, Eindhoven University of Technology, Den Dolech 2, 5600 MB Eindhoven, The Netherlands.
}

of LTE have been obtained from models which account for the effects of diffusion, excitation, ionization and recombination $[2,13,14]$.

With typical values for an argon arc plasma at one atmosphere, with an electron temperature of $1 \mathrm{eV}$, these criteria result in a critical electron density ranging from $6 \cdot 10^{22} \mathrm{~m}^{-3}$ [13] up to $10^{25} \mathrm{~m}^{-3}[2]$.

These numbers demonstrate that the theoretically determined criteria for LTE are strongly dependent on the point of departure in the reasoning. It is obvious that the value of the electron temperature is very important for the occurrence of LTE. This requires a detailed further evaluation with emphasis on acquiring additional experimental data on the deviation from LTE. The experimental study of LTE must be based on an accurate determination of electron temperature and electron density. In general, measured electron temperatures and densities at certain gas pressures have to be compared with values which follow from calculations of the plasma composition assuming LTE at that pressure [15-18].

We have followed this approach, assuming that the argon plasma studied has already reached a PLTE state in which the populations of all the energy states of the neutral spectrum, with exception of the ground state, are in thermal equilibrium with the free electrons. This assumption will be motivated in Section 2, where we describe a theoretical model for the deviation from equilibrium of the excited states of the plasma for the argon neutral spectrum. 
The electron temperature follows from the determination of the source function of suitable lines, i.e. the ratio of the emission- and the absorption coefficients of these lines [19-21] and will be discussed in Subsections 3.1. -3.4 .

The electron density follows from the measured population densities and the Saha relation; as a check, also an interferometric method has been applied, cf. Subsection 3.5. Section 4 describes briefly the experimental set-up; results of the measurements are found in Sect. 5, and Sect. 6 contains the conclusions of the present work.

\section{Equilibrium Considerations for the Argon Neutral System}

\subsection{Introduction}

An elaborate study of the overpopulation of several excited levels of helium, neon, argon and krypton systems has been published by Uhlenbusch et al. [18], and in this context helium has received by far the greatest attention. This is not so surprising as one of the major causes of overpopulation of the ground level, inward diffusion, is very pro- portant factor when a more precise determination of the electron temperature from line intensity measurements is aimed at.

In this section we use a simplified collisionalradiative model of the argon neutral system, ArI, to determine population densities of the excited levels. Our aim is not to present an exhaustive description of all the contributing processes, but rather to illustrate the influence of the more important processes on the distribution of the population densities.

\subsection{The Collisional-Radiative Model and the Validity of PLTE}

We consider the particle balance equation in state $q$. For labeling of the excited levels we use the convention $N>r>q>p>1$. The symbol $N$ identifies the maximum level which needs to be considered, $r, q$ and $p$ are the levels to be summed up, as is sketched in Figure 2.1. In Table 2.1 the used symbols are explained.

The net source term of the mass balance for the population density of level $q$

$$
\frac{\partial n_{q}}{\partial t}+\operatorname{div}\left(n_{q} \cdot \boldsymbol{w}_{q}\right) \equiv\left(\frac{\partial n_{q}}{\partial t}\right)_{C R}
$$

can be written as

$$
\begin{aligned}
& \left(\frac{\partial n_{q}}{\partial t}\right)_{C R}=n_{\mathrm{e}}\left[\sum_{p=1}^{q-1}\left(n_{p} k_{p q}-n_{q} k_{q p}\right)-\sum_{n=q+1}^{N}\left(n_{q} k_{q r}-n_{r} k_{r q}\right)-n_{q} k_{q+}+n_{\mathrm{e}} n_{1}^{+} k_{+q}^{(3)}\right] \\
& \text { exc.-deexc. from below exc.-deexc. electron 3-part. recom- } \\
& \text { from above ion bination } \\
& +n_{\mathrm{e}} n_{1}^{+} k_{+q}^{(2)} \Lambda_{+q}^{(2)}-\sum_{p=1}^{q-1} n_{q} A_{q p} \Lambda_{q p}+\sum_{r=q+1}^{N} n_{r} A_{r q r q} . \\
& \text { rad. rec. spont. emission cascade rad. }
\end{aligned}
$$

nounced in helium. In argon it is usually assumed beforehand that the deviation from equilibrium of the higher excited levels is negligible [17, 20, 21]. The ground level is generally overpopulated with respect to the excited levels, at least for ionizing plasmas. So we deal with the PLTE condition. For lower values of the temperature even the excited states are not necessarily in equilibrium with the ground state of the next ionization stage [22].

For a general approach the assumption of PLTE seems to be justified. However, the non-equilibrium population of the excited levels becomes an im-
Table 2.1. Symbols used in (2.2).

\begin{tabular}{ll}
\hline$n_{p}$ & population density of state $p\left(\mathrm{~m}^{-3}\right)$ \\
$n_{1}^{+}$ & ion ground level density $\left(\mathrm{m}^{-3}\right)$ \\
$k_{p q}$ & electron excitation rate $p \rightarrow q\left(\mathrm{~m}^{3} \mathrm{~s}^{-1}\right)$ \\
$k_{q+}$ & electron ionization rate from level $q\left(\mathrm{~m}^{3} \mathrm{~s}^{-1}\right)$ \\
$k_{+q}^{(3)}$ & three particle recombination rate coefficient $\left(\mathrm{m}^{6} \mathrm{~s}^{-1}\right)$ \\
$k_{+q}^{(2)}$ & radiative recombination rate coefficient $\left(\mathrm{m}^{3} \mathrm{~s}^{-1}\right)$ \\
$\Lambda_{+q}^{(2)}$ & escape factor for recombination radiation \\
$A_{q p}$ & transition probability for transitions state $q$ to state $p$ \\
$\Lambda_{q p}$ & escape factor for line radiation of line $q \rightarrow p$ \\
$\boldsymbol{w}_{q}$ & drift velocity of particles in state $q$. \\
\hline
\end{tabular}




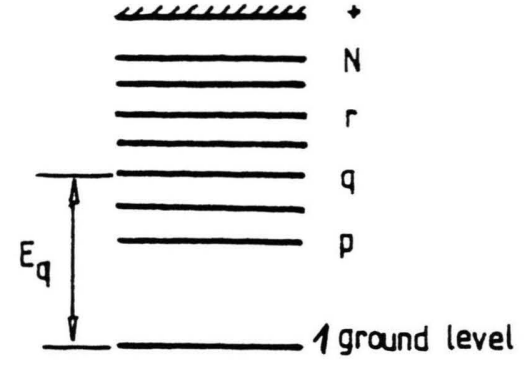

Fig. 2.1. Schematic representation of the distribution of excited levels in the energy spectrum with the notation used in this subsection.

Using the principle of detailed balancing, (2.2) can be reduced to a simpler expression, which is especially valuable for situations close to (P)LTE. We introduce here the reduced density $b_{q}$ of level $q$ defined as the ratio of the actual density $n_{q}$ and the Saha-density $n_{q \text {, Saha }}$ given by $(2.4 \mathrm{a})$, and the related relative deviation $\delta b_{q}$ through

$$
b_{q}=\frac{n_{q}}{n_{q, \text { Saha }}} \quad \text { and } \quad \delta b_{q}=b_{q}-1 .
$$

Using the Saha and Boltzmann expressions, which are only functions of the electron temperature, (2.2) can be expressed in terms of the density $n_{q \text {, Saha }}$ and relative deviation $\delta b_{q}$ as

$$
\begin{aligned}
\frac{1}{n_{q, \text { Saha }}}\left(\frac{\partial n_{q}}{\partial t}\right)_{C R}= & n_{\mathrm{e}}\left\{\sum_{i=1}^{N}\left(\delta b_{i}-\delta b_{q}\right) k_{q i}-k_{q+} \delta b_{q}\right\} \\
& +S_{q+} k_{+q}^{(2)} \Lambda_{+q}^{(2)}-\sum_{p=1}^{q-1}\left\{1+\delta b_{q}\right\} A_{q p} \Lambda_{q p} \\
& +\sum_{r=q+1}^{N}\left(1+\delta b_{r}\right) B_{r q} A_{r q} \Lambda_{r q}, \quad \text { (2.4) }
\end{aligned}
$$

where $S_{q+}$ and $B_{r q}$ are the Saha respectively the Boltzmann expressions defined as

$$
\begin{gathered}
S_{q+}\left(T_{\mathrm{e}}\right)=\frac{n_{\mathrm{e}} n_{1}^{+}}{n_{q, \text { Saha }}}=\frac{2 g_{1}^{+}}{S_{q}}\left\{\frac{2 \pi m_{\mathrm{e}} k T_{\mathrm{e}}}{h^{2}}\right\}^{1.5} \\
\cdot \exp \left\{-\frac{E_{01}-E_{q}-\Delta E}{k T_{\mathrm{e}}}\right\}
\end{gathered}
$$

and

$$
B_{r q}=\frac{n_{r, \text { Saha }}}{n_{q, \text { Saha }}}=\frac{g_{r}}{g_{q}} \cdot \exp \left\{-\frac{E_{r}-E_{q}}{k T_{\mathrm{e}}}\right\} .
$$

Here

$g_{1}^{+} \quad$ statistical weight of the ion ground level

$g_{q} \quad$ statistical weight of the excited level $q$

$E_{q} \quad$ the energy of $q$

$E_{01}=15.759 \mathrm{eV}$, the ArI ionization energy

$\Delta E=\left(2.95 \cdot 10^{-11} \mathrm{eV} \mathrm{m}^{+3 / 2} \mathrm{~K}^{1 / 2}\right) \sqrt{n_{\mathrm{e}} / T_{\mathrm{e}}}$, the reduction of the ionization energy [23].

From (2.4) the non-equilibrium population of the different excited levels can be calculated. This set of coupled equations is solved for a limited number of levels, since we only want to illustrate the applicability of PLTE. In this simplified model of the argon levels, shown in Fig. 2.2, we consider all the sublevels of one group, e.g. $4 \mathrm{~s}$, as one effective level and consequently use the effective level scheme described. This is justified because of the strong coupling existing between these sublevels $[24,25]$.

Application of the numerical values for the effective energies, statistical weights and other relevant quantities, gives the possibility to derive expressions for $\delta b_{1}, \delta b_{2}$ and $\delta b_{3}$, assuming that $\delta b_{4}=0$.

Since the collisional processes are faster than the radiative processes, nearly all excitation from the ground level by electron collisions will finally lead to ionization. In this concept the disturbance of the population distribution of excited levels as a consequence of line radiation and radiative recombination to excited levels will be restored immediately by electron excitation, leading again to ionization. The diffusion of the excited atoms can be neglected and the results for $\delta b_{2}$ and $\delta b_{3}$ as functions of the overpopulation factor of the ground state $\delta b_{1}$, are

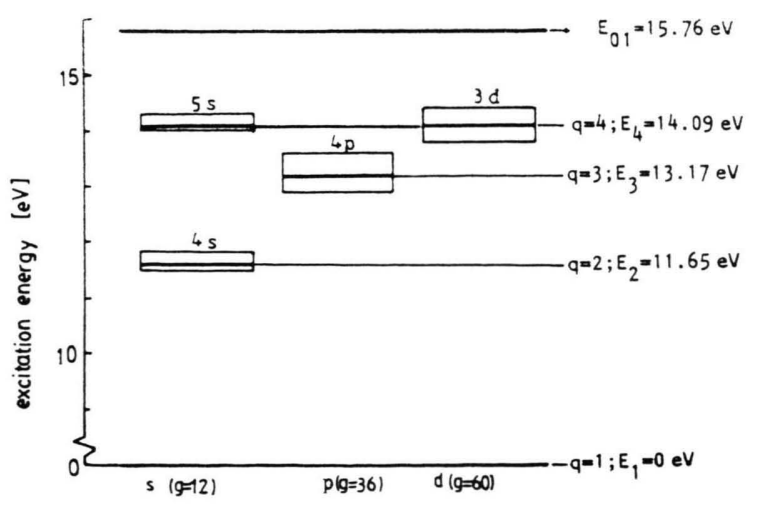

Fig. 2.2. Simplified diagram of the considered ArI excited levels showing the relation with our four level model. 
for an argon plasma with a temperature of $1 \mathrm{eV}$ (see for more details [25]),

$$
\begin{aligned}
& \delta b_{2} \approx 10^{-3} \delta b_{1}+\frac{10^{19} \mathrm{~m}^{-3}}{n_{\mathrm{e}}}, \\
& \delta b_{3} \approx 10^{-3} \delta b_{1}-\frac{5 \cdot 10^{19} \mathrm{~m}^{-3}}{n_{\mathrm{e}}} .
\end{aligned}
$$

From these equations it appears that nonequilibrium of the levels $q=2$ ( 4 s group) and $q=3$ ( $4 \mathrm{p}$ group) is mainly caused by the competition between overpopulation due to collisional (de)excitation and underpopulation due to radiative losses. In anticipation the discussion in Sect. 5, we can already state that for the parameter range of interest $\left(5 \cdot 10^{22}<n_{\mathrm{e}}<2 \cdot 10^{23} \mathrm{~m}^{-3}\right), \delta b_{2}$ will remain positive, which means that level $q=2$ will be slightly overpopulated. This overpopulation is very small, a few parts in a thousand, and is of negligible influence on our measurements. The level $q=3$ is slightly underpopulated, mainly due to the strong radiative decay to the slightly overpopulated level $q=2$.

We conclude that PLTE is indeed justified provided that $\delta b_{1}$ remains sufficiently small. A second conclusion is that in the lowest temperature region a slight underestimation of the temperature will result from our measurements if we neglect the non-equilibrium of the levels $q=2$ and $q=3$.

\subsection{Non-Equilibrium of the Ground State Level}

For ground state atoms, the diffusion can not be neglected. For a stationary argon plasma in the four level description, from (2.4) $\delta b_{1}$ can be written as $[24,25]$

$$
\begin{aligned}
\delta b_{1}=\left\{S_{1+} k_{+1}^{(2)} \Lambda_{+1}^{(2)}+\right. & B_{21} A_{21} \Lambda_{21}+B_{41} A_{41} A_{41} \\
& \left.-\frac{\operatorname{div}\left(n_{1} \cdot \boldsymbol{w}_{1}\right)}{n_{1, \text { Saha }}}\right\} / n_{e} K_{1} .
\end{aligned}
$$

Here

$S_{1+} \quad$ Saha expression for the ground state, see (2.4a).

$k_{+1}^{(2)} \quad$ radiative recombination rate coefficient, see Table 2.1,

$\Lambda_{+1}^{(2)}$ escape factor for recombination radiation, see Table 2.1,

$\boldsymbol{w}_{1} \quad$ diffusion velocity of the particles in the state $q=1$, see $(2.1)$, $n_{1, \text { Saha }}$ the Saha density, see (2.4a),

$\Lambda_{21}, \Lambda_{41}$ line escape factors,

$A_{21}, A_{41}$ effective transition probabilities for the levels $2 \rightarrow 1$ and $4 \rightarrow 1$,

$B_{21}, B_{41}$ the Boltzmann factors, see $(2.4 \mathrm{~b})$.

$K_{1}=\sum_{i=2}^{N} k_{1 i}+k_{1+}$.

Rosado [24] concluded that for the plasma parameters of interest, the radiative recombination term is the dominant radiative contribution in (2.6). The escape factor for recombination radiation appears to be in the range $0.1-0.7$ [26], whereas the escape factors of the resonant lines are of the order of $10^{-3}-10^{-2}$. The ratios of the resonant radiation of the 2-1 and 4-1 transition of the four level model to the recombination radiation are about $20 \%$ and $5 \%$, respectively. Nevertheless, we have taken these transitions into account in the model of $\delta b_{1}$.

For the conditions of interest, the calculation of the reabsorption of recombination radiation by the ground state as a function of the radius imposes a serious problem. In the spectral range above $200 \mathrm{~nm}$ hardly any absorption appears, but below $200 \mathrm{~nm}$ and specially in the wavelengths below the freebound edge $(78 \mathrm{~nm})$, the radiation is strongly absorbed, because the mean free path for photoionization is smaller than the radius of the arc [26]. Thus the reabsorption of the recombination radiation is appreciable but not large enough to allow fully for a local description by the introduction of a locally defined escape factor.

To cope with this problem we used a calculation of Hermann [26], who analysed an argon arc of $5 \mathrm{~mm}$ diameter at atmospheric pressure. Under the assumption of LTE and neglecting the effect of continuum radiation with wavelengths above the free-bound edge, he obtained radially resolved values of the net radiative recombination and consequently of the radial dependency of the escape factor. Hermann's result of the escape factor of the recombination radiation are represented in Fig. 2.3. In Fig. $2.3 \mathrm{a}$ the escape factor is given as a function of the temperature for the arc axis; Fig. $2.3 \mathrm{~b}$ gives the reduced value as a function of the radius.

In this description the reabsorption is described by a locally defined escape factor which is positive at the centre of the arc and negative in the periphery of the arc, cf. Figure 2.3. The physical meaning of a negative escape factor of the recombination radi- 

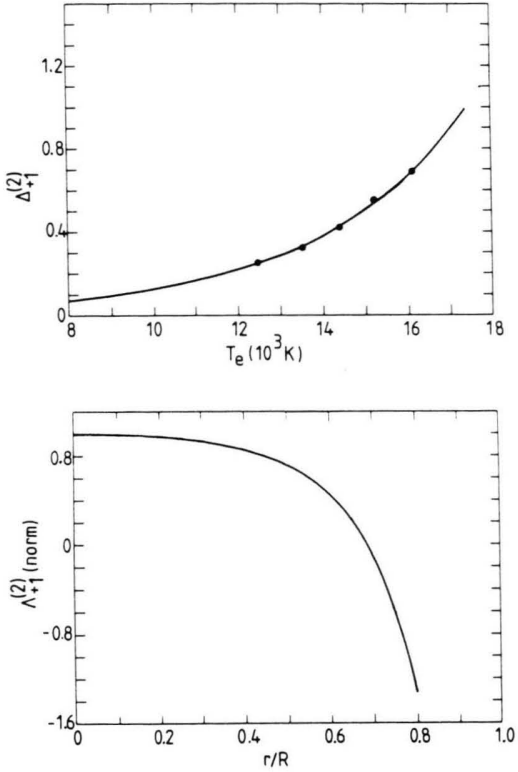

Fig. 2.3. The escape factor for recombination radiation, (a) as a function of the electron temperature for the axis $r=0 \mathrm{~mm}$, (b) reduced radial dependence of $\Lambda_{+}^{(2)}$ for a $5 \mathrm{~mm}$ diameter arc at atmospheric pressure [26]

ation is that the photoionization in the periphery, because of radiation generated more inward in hotter plasma regions, is larger than the local radiative recombination due to the decrease in the electron density. This local description has the drawback that the value of $\Lambda_{+1}^{(2)}$ is dependent on position. We will see that the diffusion effects dominate anyway in the periphery of the plasma, so the errors introduced by this procedure are small. At the center of the plasma, the influence of the diffusion becomes smaller for larger values of the current as the radial profiles flatten and radiative recombination increases because of the increase of the electron density.

\section{Principles of Measurements}

\subsection{Introduction}

This section describes the used plasma diagnostic methods. $T_{\mathrm{e}}$ is determined from the ratio of the population densities of two excited levels of the neutral-atom spectrum of argon, ArI. From relative intensity measurements of the absorption the density of the lower state is known. Together with the quasi-neutrality condition, $n_{\mathrm{e}}$ can be determined with the Saha equation. This is treated in Subsection 3.2. In Subsects. 3.3 and 3.4 the calculation and the accuracy of these measurements will be discussed. In Subsect. 3.5 the independent measurement of the electron density by means of feed-back interferometry is described.

\subsection{Determination of $T_{\mathrm{e}}$ and $n_{\mathrm{e}}$ by the Source Function Method}

The cascade arc plasma can be considered as a rotationally symmetric and axially homogeneous radiator of radius $R$ and length $l$. The construction of the cascade arc allows end-on observations, eliminating the need for Abel inversion of the measured profiles. Integrating of the radiative transfer equation over the length $l$ of the plasma column gives the intensity $I_{\lambda}(r, l)$ at a certain wavelength $\lambda$.

Under the verified assumption of PLTE combined with the black body radiation and Kirchhoff's law, one can derive that

with

$$
S_{\lambda}(r)=\frac{I_{\lambda}(r, l)}{1-\exp [-\varkappa(\lambda, r) l]}
$$

$$
S_{\lambda}=\frac{\varepsilon_{\lambda}}{\chi(\lambda)}=\frac{2 h c^{2}}{\lambda^{5}} \cdot \frac{1}{\exp \left[h c / \lambda k T_{\mathrm{e}}\right]-1}
$$

where the atomic constants $h, c$ and $k$ have their usual meaning, and where $\varepsilon_{\lambda}(r)=\varepsilon_{\lambda, l}(r)+\varepsilon_{\lambda, c}(r)$ in $\mathrm{W} / \mathrm{m}^{4} \mathrm{sr}$ and $\chi(\lambda)=x_{l}(\lambda)+\chi_{c}(\lambda)$ in $\mathrm{m}^{-1}$ are the emission and absorption coefficients, each consisting of line $l$ and continuum $c$ contributions. The ratio $\varepsilon / \varkappa$ is generally known as the source function $S_{i}[19]$.

For the determination of $T_{\mathrm{e}}$ with (3.1), besides the absolute measurement of the intensity $I_{\lambda}$, knowledge of the optical depth is necessary. The value of the optical depth can be calculated from the absorption, which is measured by imaging the arc in itself with a concave mirror behind the arc plasma. Measurements of $T_{\mathrm{e}}$ in this way eliminates the need for a transition probability in the calculations. On the other hand, only sufficiently absorbed lines come into consideration for this method [24, 25]. In our measurements, the source function is determined in a wavelength interval that includes a specific spectral line of the argon spectrum and its adjacent continuum. 


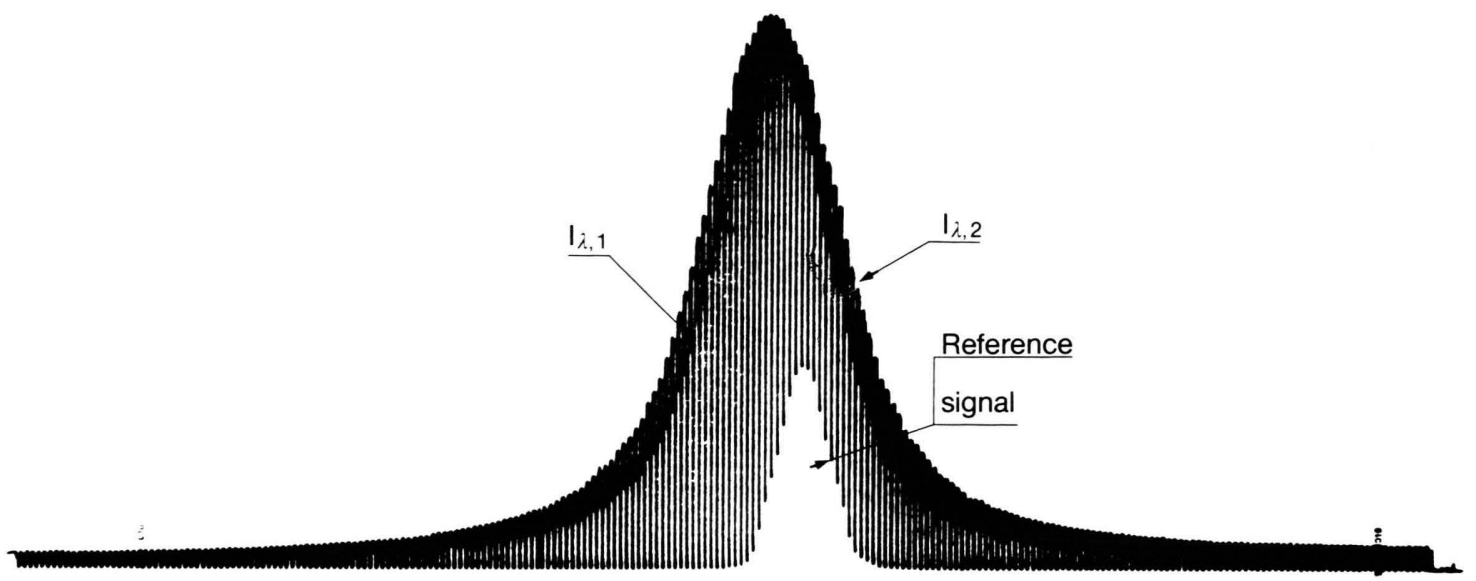

Fig. 3.1. Scan of the profiles of $I_{\lambda, 1}, I_{\lambda, 2}$ and the reference signal for the $8 \mathrm{~mm}$ argon, $I=80 \mathrm{~A}, \lambda=696.5 \mathrm{~nm}$. The scanning in this figure is from right to left $(696.0 \mathrm{~nm} \rightarrow 697.1 \mathrm{~nm})$.

Figure 3.1 shows a typical scan of the measured emission profile $I_{\lambda, 1}$ and the absorption profile $I_{\lambda, 2}$.

Additionally we measured a reference signal of a low pressure, low current argon arc discharge which is unshifted in wavelength since the electron and neutral densities are low. So, these signals can be used as an independent wavelength references for the lines from the atmospheric arc and also as the apparatus profile of the monochromator.

Some remarks on the shape of the measured profiles $I_{\lambda, 1}$ and $I_{\lambda, 2}$ denoted by $\overline{I_{\lambda, 1}^{\mathrm{m}}}$ and $\overline{I_{\lambda, 2}^{\mathrm{m}}}$ are:

1. The line profile of $\varkappa(\lambda)$ is composed of the combined effect of Stark and Doppler broadening in the plasma $[2,27]$. The Doppler contribution (Gaussian shape) to the total profile width is small compared with the contribution due to the Stark effect (Lorentzian shape), but will not be neglected. At $\lambda=700 \mathrm{~nm}$ and $T_{\mathrm{e}}=1 \mathrm{eV}$ the Doppler width is about $8 \cdot 10^{-3} \mathrm{~nm}$ and the Stark width about $50 \cdot 10^{-3} \mathrm{~nm}$. The line profile of $x(\lambda)$ is a Voigt profile with a small value of the ratio of Gaussian vs. Lorentzian widths.

2. Because the line absorption is larger at the line center than in the line-wings, the line profiles of $I_{\lambda, 1}$ and $I_{\lambda, 2}$ are different from that of $\varkappa(\lambda)$.

This is expressed by the factor $1-\exp [-\chi(\lambda) l]$.

3 . Broadening effects arising from the convolution with the apparatus profile of the monochromator are taken into account. The apparatus width is about $20 \cdot 10^{-3} \mathrm{~nm}$ and becomes an important contribution to the profiles measured at low values of the arc current.
Measurement of the line-profiles of $I_{\lambda, 1}$ and $I_{\lambda, 2}$ allows to determine also other plasma parameters besides $S_{\lambda}$ (and thus $T_{\mathrm{e}}$ ), e.g. the electron density $n_{\mathrm{e}}$. This $n_{\mathrm{e}}$-value follows from the combination of the integrated value of $x(\lambda)$, which yields the lower excited state density $n_{1}$ and the Saha equation. In this case the relevant transition probability is needed. The Saha-equation contains $n_{1}, n_{\mathrm{e}}$, the ion density $n_{\mathrm{i}}$ and the measured value $T_{\mathrm{e}}$. With the quasineutrality condition $n_{\mathrm{e}}=n_{\mathrm{i}}, n_{\mathrm{e}}$ can be calculated. Furthermore the density of the ground state atoms $n_{1}$ is determined from Dalton's law

$$
p=\left(2 n_{\mathrm{e}}+n_{1}\right) k T_{\mathrm{e}},
$$

where $p$ is the pressure.

Because we also want to determine $\delta b_{1}$, we need the Saha value of the neutral density $n_{1, \text { Saha }}$. This value follows directly from $(2.4 \mathrm{a})$ with the experimental values of $T_{\mathrm{e}}$ and $n_{\mathrm{e}}$.

\subsection{Calculation Procedure for the Plasma Parameters}

We have used a numerical procedure in which values of the relevant plasma parameters were calculated iteratively from a comparison of the theoretically predicted intensity distributions $I_{\lambda, 1}$, and $I_{\lambda, 2}$ with the measured intensity distributions

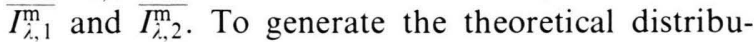
tions $I_{\lambda, 1}$ and $I_{\lambda, 2}$ in the numercial procedure, one should take into account:

1. The Lorentzian component of the profile $x_{1}(\lambda)$ due to the Stark effect caused by the electron density. 
2. The Gaussian component of $\chi_{1}(\lambda)$ due to the Doppler effect.

3. The influence of the continuum $x_{\mathrm{c}}(\lambda) l$ with the $\xi$-factor for the free-free emission taken from [31, 32].

The line profile of $x_{1}(\lambda) l$ is thus a Voigt function which can be calculated from the real part of the complex error function [33], using the fast algorithm developed by Gautschi [34]. With $\chi(\lambda) l$ and the value of $T_{\mathrm{e}}, I_{\lambda, 1}$ and $I_{\lambda, 2}$ are constructed.

The influence of the apparatus profile is accounted for by convolution of the theoretical intensities profiles with the measured apparatus profile $A(\lambda)$.

The fit to the measured profiles was performed with a least-squares minimization procedure [28] in which the Marquardt algorithm was used [29]. In this procedure the constructed intensity distributions $\overline{I_{\lambda, 1}}=I_{\lambda, 1} \cdot A(\lambda)$ and $\overline{I_{\lambda, 2}}=I_{\lambda, 2} \cdot A(\lambda)$ are compared with the measured distributions $\overline{I_{\lambda, 1}^{\mathrm{m}}}$ and $\overline{I_{\lambda, 2}^{m}}$ for each wavelength value.

\subsection{The Accuracy of the Plasma Parameters}

The accuracy of the absolute calibration of the measured intensity, done with a calibrated tungsten ribbon lamp (Philips T234 type W2kGV22i) influences the various parameters. The emissivity for a strip temperature of $2600 \mathrm{~K}$ can be found from the tables given by de Vos [30]. The relative accuracy of the calibration is about $3 \%$. It is obvious that an error in the calibration factor leads to errors in

1. the source function and consequently also in the temperature,

2. the electron density, which is on its turn coupled via the Saha equation with the temperature and the excited level density.

ad 1: The relative error in the source function is equal to the relative error in the calibration factor. From (3.1) it can be derived that with a relative error in the calibration of about $3 \%$, the error in the temperature is about $2 \%$.

ad 2: The error in the determination of the electron density is determined by two effects:

a) The error in the excited state density $n_{1}$ which is calculated from the optical depth, which value is affected by the inaccuracy of the relative measurement of the emission and absorption, the influence of the transmittance of the windows of the arc and the reflectance of the mirror BM, see Figure 4.1.
Additionally also the uncertainties in the arc length $l$ and the transition probability $A_{\mathrm{u} 1}$ in the optical depth lead to an estimation of about $5 \%$.

b) The error in the electron temperature, which influences the determination of the electron density through the Saha-equation. For the relative error in the electron density we obtain

$$
\frac{\Delta n_{\mathrm{e}}}{n_{\mathrm{e}}}=\left\{\frac{3}{4}+\frac{\gamma}{2 T_{\mathrm{e}}}\right\} \cdot \frac{\Delta T_{\mathrm{e}}}{T_{\mathrm{eo}}}+\frac{\Delta n_{l}}{2 n_{l}},
$$

where $\gamma=\left(E_{01}-\Delta E-E_{q}\right) / k \approx 4.78 \cdot 10^{4} \mathrm{~K}$ for a $4 \mathrm{p}-4 \mathrm{~s}$ transition.

The error in the electron density over the temperature range 10000 up to $15000 \mathrm{~K}$ is about $10 \%$.

Additionally it can be remarked that the functional relationship between $\Delta n_{\mathrm{e}} / n_{\mathrm{e}}$ and $\Delta T_{\mathrm{e}} / T_{\mathrm{e}}$ is such that the variation in the values of $\Delta n_{\mathrm{e}} / n_{\mathrm{e}}$ as a function of $T_{\mathrm{e}}$ has practically the same slope as the $T_{\mathrm{e}}-n_{\mathrm{e}}$-relation. This important conclusion we will discuss in Section 5.

\subsection{Interferometric Determination of the Electron Density}

For the determination of number densities, interferometric methods have the advantage of being independent of any assumption of (P)LTE and are based on the determination of changes in the index of refraction of a plasma. We used an interferometer of coupled-cavity type as first described by Ashby and Jephcott [35], and have been probing the same plasma volume as with the spectroscopic set-up [36].

Due to the high density of the neutrals their contributions to the index of refraction cannot be ignored. So, a two wavelength interferometer, viz. a $\mathrm{He}-\mathrm{Ne}$ laser, with wavelengths of $632.8 \mathrm{~nm}$ and $3390 \mathrm{~nm}$ is used. To obtain values of the electron density in the stationary state from the variation in the refractive index, the arc is short-circuited. In the afterglow usually the electron density decay is faster than the increase of the neutrals, and the variation in the electron density can be determined with more precision than the variation of the neutrals.

\section{Experimental Set-Up}

The spectroscopic set-up consist of a telecentric optical system with a spatial resolution of $0.4 \mathrm{~mm}$ and an acceptance angle of about $1 \mathrm{mrad}$ which 


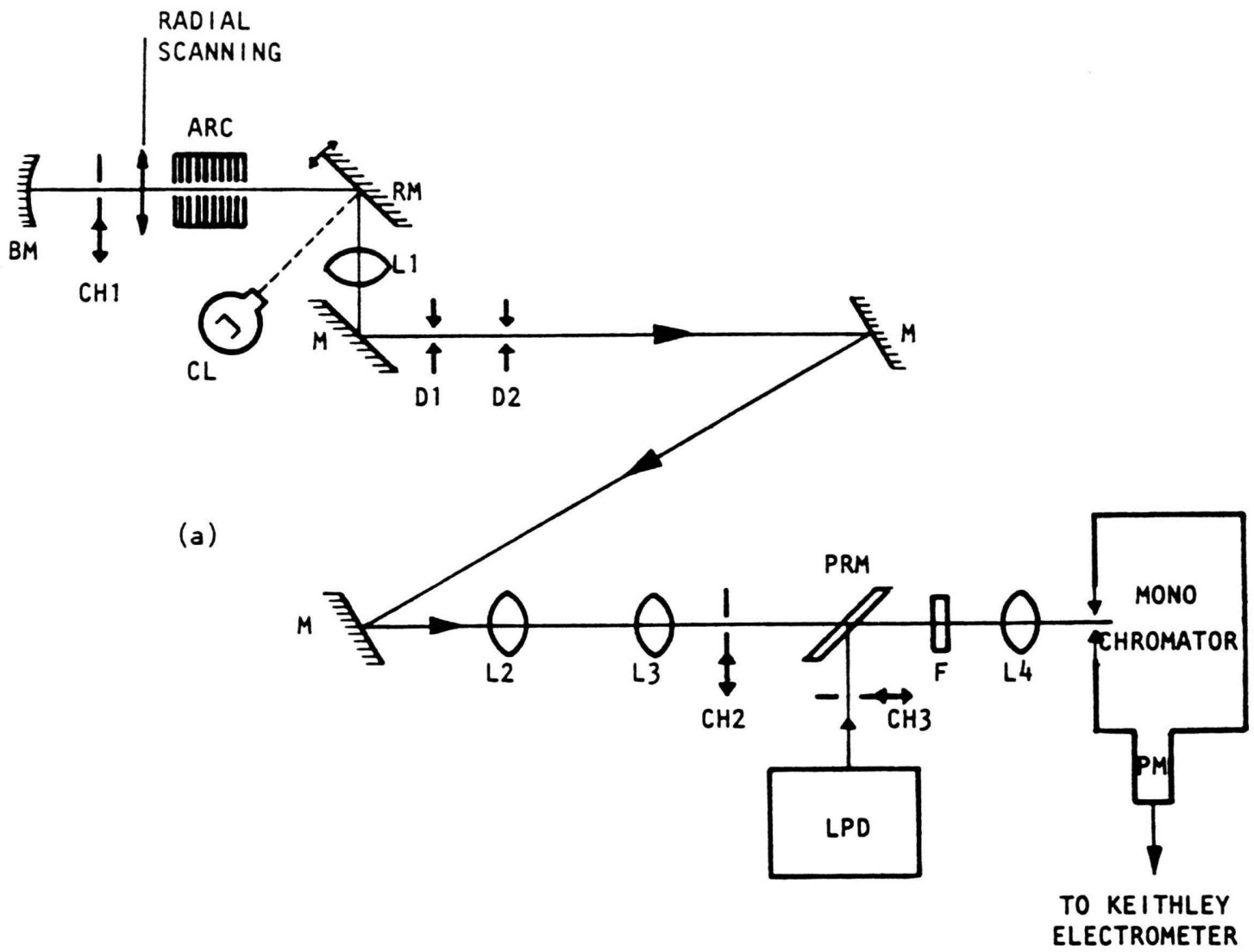

Fig. 4.1. Diagram of the spectroscopic set-up and data acquisition system. L 1, L2, L 3 and L 4: lenses, D 1 and D2: pinholes of $0.4 \mathrm{~mm}$ diameter, $\mathrm{CH} 1, \mathrm{CH} 2$ and $\mathrm{CH} 3$ : computer controlled choppers, $\mathrm{RM}$ : movable mirror, BM and $\mathrm{M}$ : mirrors, PRM: 50\% transoport mirror, LPD: low pressure argon discharge, F: filter, PM: photomultiplier type EMI $9698 \mathrm{QB} / \mathrm{S} 20$.

images the plasma on the slit of a Jarrell-Ash $1 \mathrm{mtr}$ double monochromator of the Czerny-Turner type, see Figure 4.1. The optical system allows also for the inclusion of the additional profile measurement acquired from the low pressure argon discharge as discussed in Section 3.2. Radial scanning of the plasma is possible by moving the arc construction planparallel to the optical axis of the optical detecting system. Scanning of the complete spectral line and its adjacent continuum with the monochromator is controlled by a DEC PDP $11 / 03$ computer. The data are sent for storage to a DEC PDP 11/23, and further processing of the data can be performed with a Burroughs B 7700 computer.

With coupled-cavity type interferometer the electron and neutral densities are detected by shortcircuiting the arc, and the data are stored in LeCroy
2256 waveform digitizers which are coupled with the PDP $11 / 03$ computer.

The plasma is produced in a cascade-arc of the "Maecker"-type with arc channel diameter of 5 or $8 \mathrm{~mm}$. The influence of the arc ends on the interferometry results is studied with two different arc lengths, viz. 58 and $84 \mathrm{~mm}$ for both arc diameters. In $[24,25]$ the experimental set-up is described in more detail.

\section{Experimental Results and Discussion}

\subsection{Survey}

In 5.2 the results of the temperature and density measurements are given and compared with the corresponding LTE values. In 5.3 the measured 
overpopulation of the ground level and the predicted one with aid of the four level model will be discussed. In 5.4 values for the total rate coefficient are derived from the results. In 5.5. the results for the electron density obtained with the source function method are compared with the results obtained with the interferometric method.

\subsection{Electron Temperature and Density}

The radially resolved $T_{\mathrm{e}}$ and the $n_{\mathrm{e}}$ were measured in arcs of two different diameters and of two different lengths at atmospheric pressure in the range of 40-200 Amps. Using the procedure described in 3.2 , the absorption and emission profiles of 5 lines of the $4 p-4 s$ group of the argon neutral spectrum were measured, viz. the 696.5, 727.3, $750.4,763.5$ and $794.8 \mathrm{~nm}$ spectral lines [37]. With the verified assumption of PLTE, $T_{\mathrm{e}}$ is determined from this set of parameters, and these values coincide within about $2 \%$, which is within the experimental error.

We conclude that further investigations of $T_{\mathrm{e}}$ can be carried out using the results of measurements for one transitions for which the $696.5 \mathrm{~nm}$ line was chosen. Radial temperature profiles are given in Fig. 5.1 for different values of the arc current.

From the total line intensity, values of $n_{\mathrm{e}}$ can be calculated using the PLTE model with $A=6.7$ $10^{6} \mathrm{~S}^{-1}$ [37] for the transition probability of the $696.5 \mathrm{~nm}$ line, see Figure 5.5. In this way for each measurement we obtain pairs of values of the $T_{\mathrm{e}}$ and $n_{\mathrm{e}}$, shown in Fig. 5.2 for 5 and $8 \mathrm{~mm}$ diameter argon arcs at atmospheric pressure. Also drawn in the figure is the $T_{\mathrm{e}}-n_{\mathrm{e}}$-relationship, that results from LTE calculations, viz. Dalton's law together

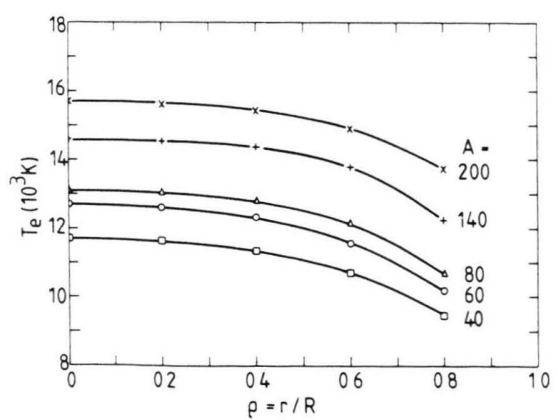

Fig. 5.1. Radial dependence of $T_{\mathrm{e}}$ for 5 values of the arc current.

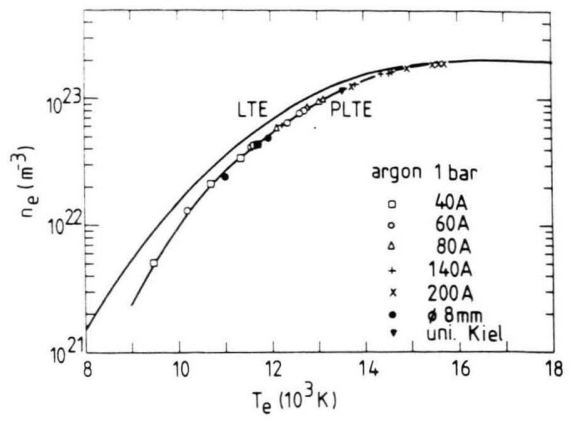

Fig. 5.2. The relation between $T_{\mathrm{e}}$ and $n_{\mathrm{e}}$ in an atmospheric argon arc plasma. PLTE: Values of $T_{\mathrm{e}}$ and $n_{\mathrm{e}}$ determined experimentally under the assumption of partial local thermal equilibrium. LTE: Values of $T_{\mathrm{e}}$ and $n_{\mathrm{e}}$ from LTE calculations of the plasma composition. The results are of measurements with $5 \mathrm{~mm}$ and $8 \mathrm{~mm}$ diameter arcs and a result obtained in a $4 \mathrm{~mm}$ diameter arc of the University of Kiel (BRD).

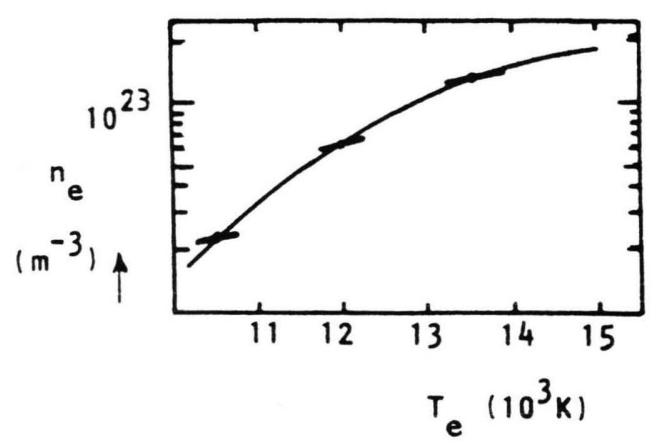

Fig. 5.3. Variation of $\Delta n_{\mathrm{e}} / n_{\mathrm{e}}$ along the measured $T_{\mathrm{e}}-n_{\mathrm{e}}$ relation for an uncertainty in $T_{\mathrm{e}}$ equal to $2 \%$.

with quasi-neutrality and the Saha-equation with the experimental electron temperature at a pressure of 1.035 bar.

At higher values of the current the measured curve approaches the LTE curve, at lower values the discrepancy becomes larger, as expected [22]. In Fig. 5.2 it is seen that the relationship between the electron temperature and density appears to be rather independent of the radial position in the arc. Also shown in Fig. 5.2 is a value obtained from a measurement on a $4 \mathrm{~mm}$ diameter atmospheric pressure argon arc, performed at the University of Kiel [38].

The data analysis was done with the numerical methods described in 3.3. We show the result of the discussion in 3.4 regarding the coupled variation of the electron density and temperature, in Figure 5.3. 


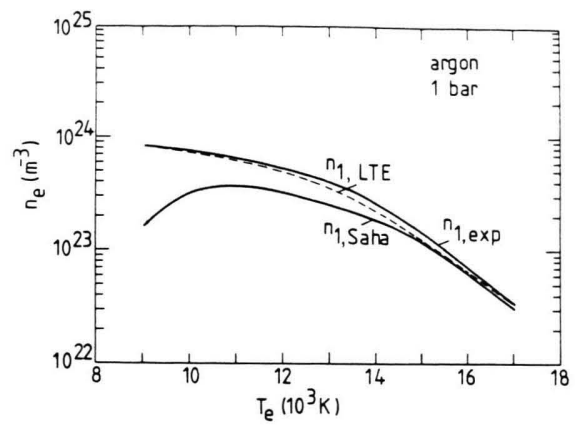

Fig. 5.4. The density of the neutral ground level as a function of the electron temperature. $n_{1 \text {,exp }}$ : measured relationship, assumption of PLTE. $n_{1, \mathrm{LTE}}$ : LTE calculation. $n_{1, \text { Saha }}:$ values from the Saha equation with experimental values of $n_{\mathrm{e}}$ and $T_{\mathrm{e}}$.

As is evident, the variations in the electron density and temperature are such that the errorbars are nearly parallel to the $T_{\mathrm{e}}-n_{\mathrm{e}}$-relation. This means that small errors introduced in the determination of the electron density from the source function method will hardly influence our comparisons between the experimentally determined $T_{\mathrm{e}}-n_{\mathrm{e}}-$ relation and the LTE relation.

As described in 3.2, with the aid of Dalton's law and together with the experimental values of the temperature and electron density, the actual plasma neutral density, $n_{1, \exp }$ can be calculated from $n_{1, \exp }=$ $p / k T_{\mathrm{e}, \exp }-2 n_{\mathrm{e}, \exp }$. This is given in Figure $5.5 \mathrm{~b}$.

The experimental values for $n_{1, \exp }$ can be compared with two formal parameters, see Figure 5.4.

a) $n_{1, \text { Saha }}$, which would be the ground state density if the ground level would be in equilibrium with the measured electron temperature and density.

b) $n_{1, \mathrm{LTE}}$, which would be the ground state density if the system was in LTE with temperature $T_{\mathrm{e}}$ at 1 atmosphere. Note that the corresponding $n_{\mathrm{e}, \mathrm{LTE}}$ differs from the actual value $n_{\mathrm{e}, \mathrm{exp}}$, cf. Figure 5.2.

There are two reasons to choose $n_{1, \text { Saha }}$ as the equilibrium quantity to compare with $n_{1, \exp }$ and to calculate from the ratio $n_{1, \exp } / n_{1 \text {, Saha }}$ the deviations from equilibrium, as expressed in the overpopulation factor $\delta b_{1}$, see (2.3).

The first and also formal reason is that this quantity appears in the expressions of the applied collisional radiative model and in the relative deviation from equilibrium, see Section 2. A second reason however is that at low temperatures the

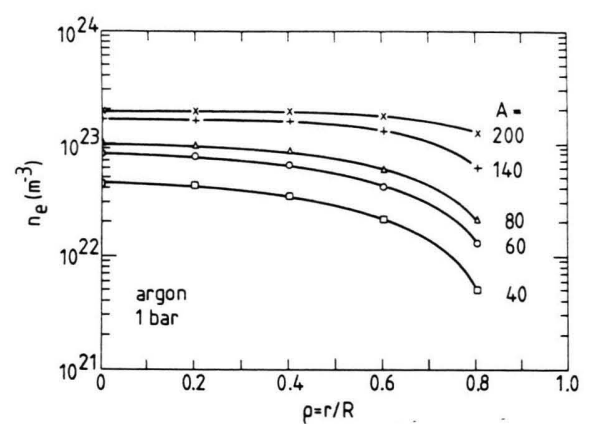

(a)

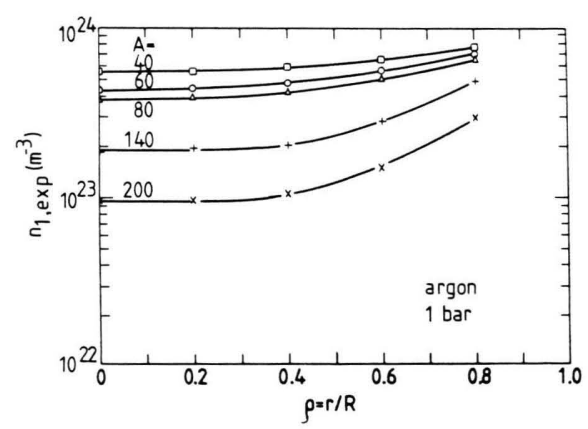

(b)

Fig. 5.5. Radial dependence of (a) the electron density $n_{\mathrm{e}, \exp }$ and (b) the neutral density $n_{1, \exp }$. Parameter is the arc current in argon by 1 bar for the $5 \mathrm{~mm}$ arc.

deviation of $n_{1, \text { exp }}$ and $n_{1, \text { LTE }}$ becomes small by the more reason that contribution of the electron density diminishes in Dalton's law. So the confluence of $n_{1, \text { exp }}$ and $n_{1, \text { LTE }}$ at low temperatures would give a false suggestion of an approach to equilibrium, whereas in fact the deviation becomes larger for the low temperature region. This can be clearly seen in Figure 5.4.

\subsection{Measured Overpopulation Factor versus Model Value}

In this subsection the measured overpopulation factor of the argon neutral ground level will be compared with the calculated overpopulation factor derived from the four level model as described by (2.6). To this end we discuss first in some more detail the diffusion and the radiative effects that contribute to the value of $\delta b_{1}$ derived from the model.

The contribution of the diffusion term $\operatorname{div}\left(n_{1}, \boldsymbol{w}_{1}\right)$ to $\delta b_{1}$ in (2.6) will be expressed in terms of $n_{\mathrm{e}}$ and $T_{\mathrm{e}}$ by using the relations

$\nabla \cdot n_{1} \boldsymbol{w}_{1}=-\nabla \cdot n_{\mathrm{e}} \boldsymbol{w}_{\mathrm{e}}=\nabla \cdot \frac{D_{\mathrm{A}}}{2 k T_{\mathrm{e}}} \operatorname{grad}\left(p_{\mathrm{e}}+p_{\mathrm{i}}\right)$, 
where $\boldsymbol{w}_{\mathrm{e}}$ is the electron diffusion velocity, $p_{\mathrm{e}}, p_{\mathrm{i}}$ are the partial pressures of resp. electrons and ions and $D_{\mathrm{A}}$ is the ambipolar diffusion coefficient, given in [39] as

$$
D_{\mathrm{A}}=2 \frac{3 k T_{\mathrm{e}}}{8\left(n_{1}+n_{i}\right) \Omega_{i, 1}^{(1,1)} m_{1}}\left[\mathrm{~m}^{2} \mathrm{~s}^{-1}\right] .
$$

Here $m_{1}$ is the heavy particle mass and $\Omega_{i, 1}^{(1,1)}$ is a first approximation to the ion-atom collision integral, which can be calculated [39] from

$$
\Omega_{i, 1}^{(1,1)}=2.84 \cdot 10^{-17} T_{i, 1}^{0,36}\left[\mathrm{~m}^{3} \mathrm{~s}^{-1}\right],
$$

where $T_{i, 1}$ is the ion temperature.

As shown by Gurevich et al. [40] and Nick et al. [22] the assumption that the ion temperature equals the electron temperature is justified for our investigated plasma conditions. With (5.3) we get for (5.2)

$$
D_{\mathrm{A}} \approx 2 \cdot \frac{2.76 \cdot 10^{18}}{n_{1}+n_{\mathrm{e}}} T_{\mathrm{e}}^{0.64} .
$$

With the measured radial profiles of $T_{\mathrm{e}}, n_{\mathrm{e}}$ and the calculated radial density profile of $n_{1, \exp }$ it is possible to estimate the influence of the diffusion term on the value of $\delta b_{1}$. The experimental values have been fitted with a least squares approximation to a bi-quadratic profile, viz.

$$
x(\varrho) / x(0)=1+b_{1} \varrho^{2}+b_{2} \varrho^{4}
$$

with $\varrho=r / R$ and $x$ the plasma parameter.

These profiles are then differentiated to yield values of the diffusion term in (2.6). In Fig. 5.6 this diffusion term is given as a function of the radius for the current range of $40-200 \mathrm{~A}$, viz.

$$
\delta b_{1}^{\bmod }(\operatorname{dif})=\frac{\operatorname{div}\left(n_{1} \boldsymbol{w}_{1}\right)}{n_{1, \text { Saha }}} \frac{1}{n_{\mathrm{e}} K_{1}^{\mathrm{th}}} .
$$

The rate coefficient $K_{1}^{\text {th }}$ is taken from a numerical collisional radiative model which includes 49 levels of the argon neutral system [41].

We will discuss now the total radiative effects which contribute to the deviation from equilibrium. This total radiative contribution can be written as, see (2.6),

$$
\begin{aligned}
& \delta b_{1}^{\text {mod }}(\mathrm{rad}) \\
& \quad=\left\{S_{1+} k_{+1}^{(2)} \Lambda_{+1}^{(2)}+B_{21} A_{21} A_{21}+B_{41} A_{41} A_{41}\right\} / n_{\mathrm{e}} K_{1}^{\text {th }}
\end{aligned}
$$

and is given in Fig. 5.7 as a function of the radius for three values of the current.

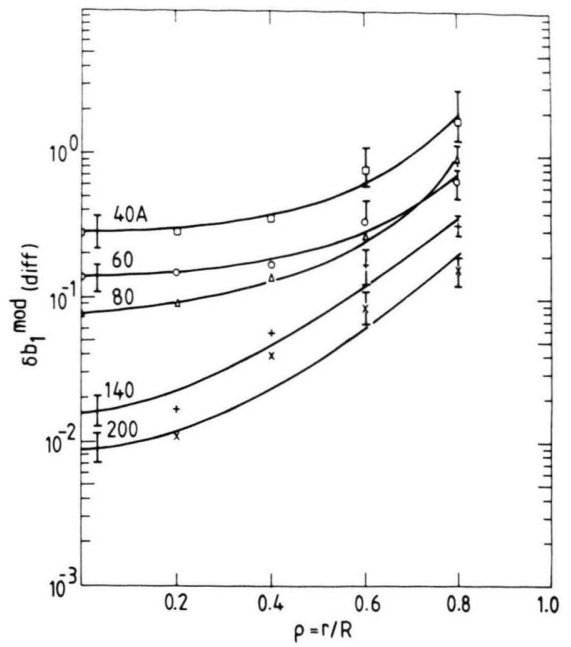

Fig. 5.6. The radial dependency of the diffusive term of (2.6).

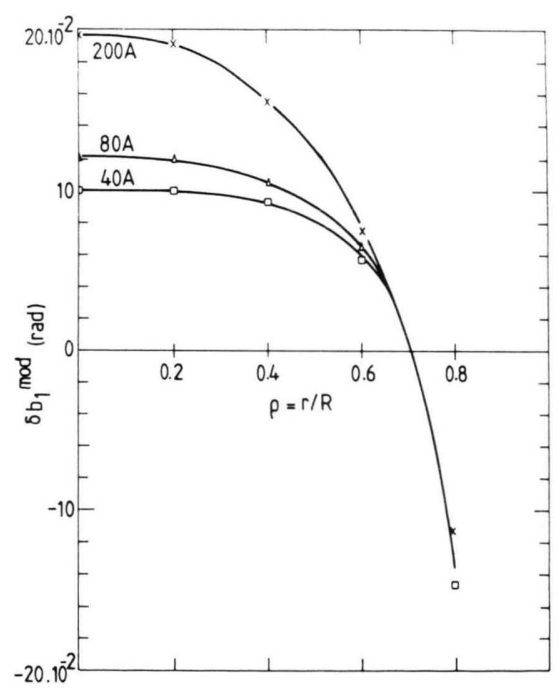

Fig. 5.7. The radial dependency of the recombination radiation and the resonant radiation terms of $(2.6)$.

Here $S_{1+}$ and $B_{21}, B_{41}$ are calculated from (2.4) with the measured $T_{\mathrm{e}}$ and $n_{\mathrm{e}}$. The recombination rate coefficient is taken from Katsonis [42] as

$$
k_{+1}^{(2)} \approx 0.995 \cdot 10^{-19} \hat{T}_{\mathrm{e}}^{-0.17} \mathrm{~m}^{3} \mathrm{~s}^{-1}
$$

with $T_{\mathrm{e}}$ in $\mathrm{eV}$. If we take into account the advance of series limit [43] as

$$
\Delta E_{\mathrm{opt}}=1.07 \hat{T}_{\mathrm{e}}-0.62 \mathrm{eV}
$$


the value $1.55 \cdot 10^{-19} \mathrm{~m}^{3} \mathrm{~s}^{-1}$ as the recombination rate coefficient can be calculated over the considered temperature range. The escape factor for recombination radiation is taken from the description of Hermann [26] as has been discussed in Section 2.3, cf. Figure 2.3.

Concerning the line escape factors $\Lambda_{21}$ and $\Lambda_{41}$, these are calculated from an extension of the treatment of Klein [44]. In this calculation it is assumed that the absorption and emission profiles can be described with Voigt profiles. The Lorentzian and Gaussian halfwidths are known from the experimental data. The escape factor can be derived from the optical depth, which is a function of these halfwidths [24]. Note that the treatment of the resonance radiation is less elaborate than that of the recombination radiation. Here the treatment is purely local and no allowance is made for the possibility of a negative escape factor to describe a net absorption at the periphery of the plasma. As is discussed in 2.3, the resonant radiation of the transitions $2-1$ and $4-1$ is about $20 \%$ respectively $5 \%$ and is of minor influence.

We will now compare the measured and the model overpopulation factor. The radial dependence of the measured overpopulation factor is shown in Fig. 5.8 for the current range 40-200 A and can be calculated as, see (2.3),

$\delta b_{1}^{\exp }=\frac{n_{1, \exp }}{n_{1, \text { Saha }}}-1 \quad$ with $\quad n_{1, \exp }=\frac{p}{k T_{\mathrm{e}, \exp }}-2 n_{\mathrm{e}, \exp }$.

Here $p$ is a pressure of $1.035 \cdot 10^{5} \mathrm{Nm}^{-2}$ and $n_{1, \text { Saha }}$ is the Saha ground level density, calculated with the experimental $T_{\mathrm{e}}$ and $n_{\mathrm{e}}$. In Fig. 5.9 this quantity is plotted as a function of $T_{\mathrm{e}}$ for the central part of the arc. The broken line is the best fit through the experimental points. For comparison, two values obtained by Bober at al. [45] have also been inserted
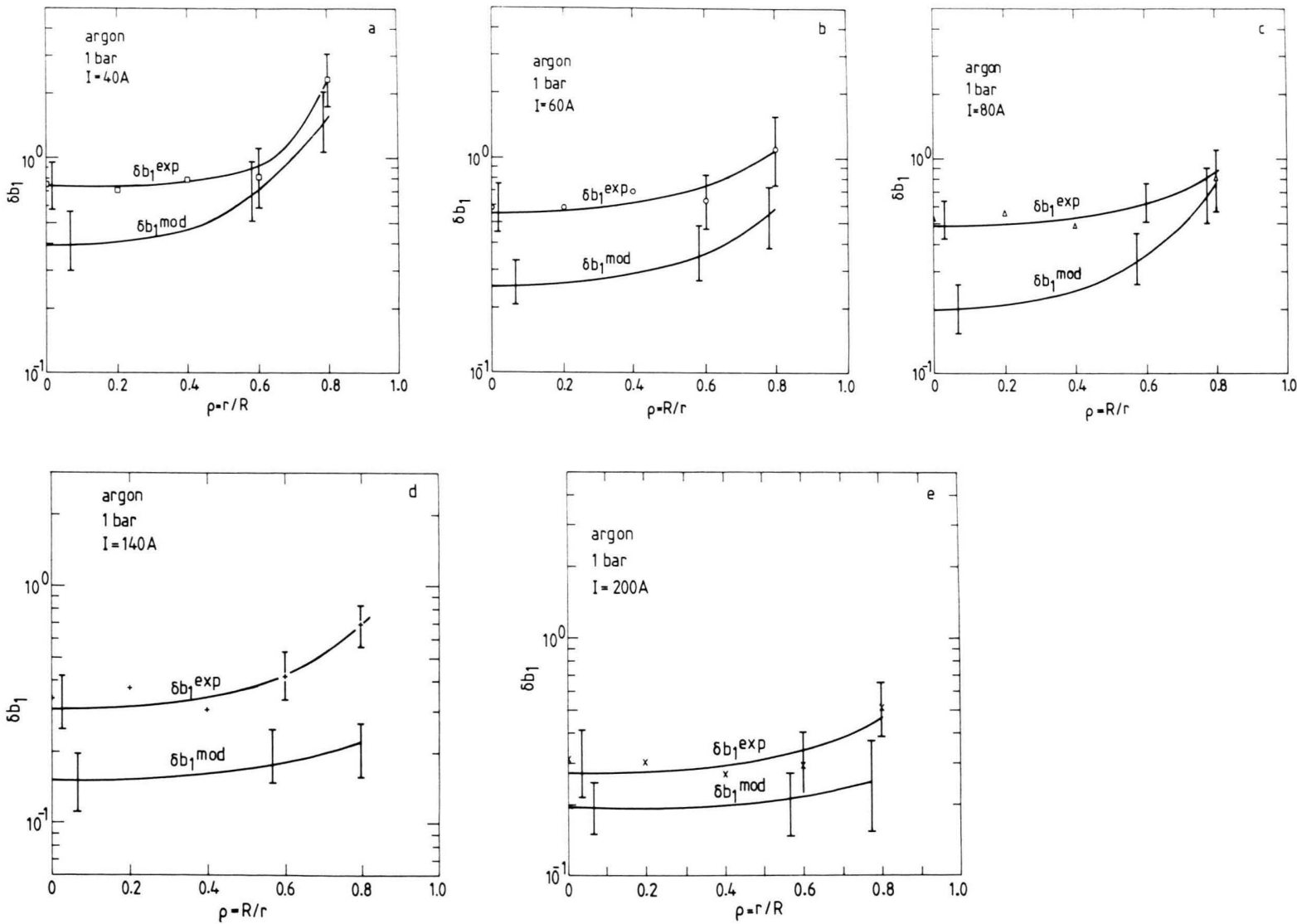

Fig. $5.8 \mathrm{a}-\mathrm{e}$. The radial dependency of the measured overpopulation factor, $\delta b_{1}^{\exp }$ of the argon neutral ground level compared to the $\delta b_{1}^{\text {mod }}$, the overpopulation factor derived from the four level model for the current range of $40-200 \mathrm{~A}$. 


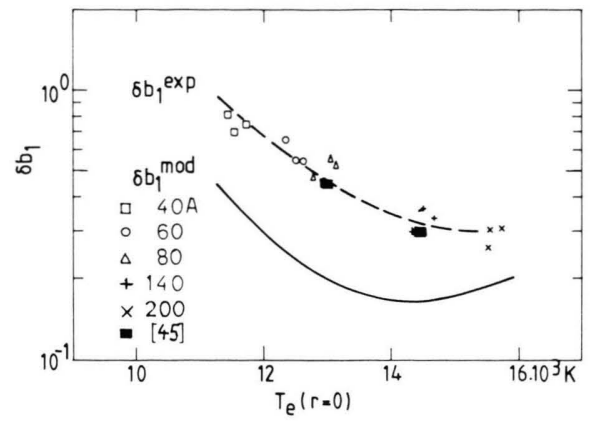

Fig. 5.9. The temperature dependency of $\delta b_{1}^{\exp }$ and $\delta b_{1}^{\text {mod }}$.

in Figure 5.9. The agreement with our experiments is good. The error bars in Fig. 5.8 are based on the experimentally obtained accuracy in $T_{\mathrm{e}}$ of $2 \%$ and in $n_{\mathrm{e}}$ of $10 \%$. These variations are not independent as has been discussed in Section 3.4.

In the Figs. 5.8 and 5.9 also the values of the overpopulation factor $\delta b_{1}^{\text {mod }}$ which follow from the four level model are shown. The error bars of the model values are based on the same variations of $T_{\mathrm{e}}$ and $n_{\mathrm{e}}$ as applied to the experimental points.

Based on the experimental overpopulation factor of the argon neutral ground level and the predicted overpopulation factor derived from the four level model the following conclusions can be drawn:

1. The radial profiles of the experimental overpopulation factor, cf. Fig. 5.8 are rather flat for $0<r / R<0.5$. In the periphery of the arc a significant increase of $\delta b_{1}^{\exp }$ occurs towards the wall, especially for the lowest current. These larger deviations from the LTE model can be expected due to the increasing effect of the diffusion caused by the steep density gradients which occur near the wall, cf. Figure 5.6.

2. For the current range of $40-60 \mathrm{~A}$ the diffusion gives the main contributions to $\delta b_{1}^{\text {mod }}$ though the total radiative contribution can not be ignored. This main diffusion influence can be expected from the gradients of the measured $T_{\mathrm{e}}$ and $n_{\mathrm{e}}$. The radiative contribution increases with increasing currents, in particular near the arc axis. In the current range of 140-200 A at the center of the arc the diffusion contributes less than the total radiation, compare Figs. 5.6 and 5.7.

3. The recombination radiation is the major radiative contribution to the overpopulation.

4. Over the whole current range and nearly over all radial positions, the value of $\delta b_{1}^{\text {mod }}$ is roughly a factor of two less than the experimental value of the deviation from equilibrium, $\delta b_{1}^{\exp }$. We want to point out here that the model underestimates the recombination radiation even though we take already the enlarged recombination rate. This is due to the mere reason that in Hermann's calculation of the escape factor of the recombination radiation [26] the LTE model is used. And as one can conclude from the experiments the plasma of the cascade arc deviates from LTE.

5. In the current range of 140-200 A the diffusive part of $\delta_{1}^{\text {mod }}$ becomes larger than the radiative part for radii larger than half the arc radius. This is due to the rather steep density gradients near the wall.

6. In 2.2 we have discussed the overpopulation factors for the levels $q=2$ and $q=3$. With the measured overpopulation $\delta b_{1}$ it can be derived that $\delta b_{2}$ and $\delta b_{3}$ are negligible in comparison with $\delta b_{1}$ and this yields a justification for the PLTE proposition.

Uhlenbusch et al. [17, 46] and Nick [47] reported about the influence of diffusion and non-equilibrium populations in noble gas plasmas. The general conclusions on the dominance of diffusion are similar. However, the results for the periphery of the plasma differ; our measurements performed up to a reduced radius of 0.8 result in positive overpopulation factors, whereas the results of Uhlenbusch and Nick at a reduced radius of 0.9 point to underpopulated ground states. Furthermore, the relation in our measurements between $T_{\mathrm{e}}$ and $n_{\mathrm{e}}$ appears to be rather independent of the radius in the arc. Uhlenbusch and Nick concluded that for reduced radii larger than 0.5 this independence is not present.

The difference between our results and the results of Uhlenbusch's as well as Nick's side-on measurements obtained at the plasma may be related to the difference in diagnostic method and arc construction. Our measurements, performed end-on, allow for very thin interspacing rings whereas Nick's side-on measurements require a larger interspacing, this may lead to a smaller influence of the diffusion effects which in our $40 \mathrm{~A}$ plasma determine the observed overpopulation factor.

With the aid of the energy balance for $40 \mathrm{~A}$ endon measurements, performed on a $4 \mathrm{~mm}$ diameter arc, Nick calculated a difference of about $200 \mathrm{~K}$ between the gas temperature and $T_{\mathrm{e}}$ at plasma conditions of $12.000 \mathrm{~K}$ and $n_{\mathrm{e}}=7 \cdot 10^{22} \mathrm{~m}^{-3}$ [22]. He 
also concluded from his measurements that for $n_{\mathrm{e}}$ less than $6 \cdot 10^{21} \mathrm{~m}^{-3}$ non-equilibrium effects have their influences on the $4 p$ level of neutral argon. This is in full agreement with our calculations of the overpopulation factors $\delta b_{2}$ and $\delta b_{3}$ derived from the four level model in which the $4 \mathrm{p}$ levels are included, cf. Chapter 2.

In spite of the difference in the periphery of the arc, the general conclusions about the overpopulations still agree well with those of both authors, especially quantitatively for the central core of the 3 different arcs.

\subsection{The Total Excitation and Direct Ionization Rate Coefficient for the Argon Neutral Ground Level}

From the measured radial values of the relative overpopulation factor $\delta b_{1}^{\exp }$ for the argon neutral ground level, $n_{\mathrm{e}}$ calculated by means of the Sahaequation using the measured $T_{\mathrm{e}}$ and the density $n_{1, \text { Saha }}$, together with the calculated diffusion flux and the radiation terms, the total excitation and direct ionization rate coefficients can be determined with (2.6) as

$$
\begin{aligned}
K_{1}^{\exp } & =\left\{S_{1+} k_{+1}^{(2)} \Lambda_{+1}^{(2)}+B_{21} A_{21} \Lambda_{21}\right. \\
& \left.+B_{41} A_{41} \Lambda_{41}-\frac{\operatorname{div}\left(n_{1}, w_{1}\right)}{n_{1, \text { Saha }}}\right\} / n_{\mathrm{e}, \exp } \delta b_{1}^{\text {exp }} .
\end{aligned}
$$

Results for $K_{1}^{\text {exp }}$ are shown in Figure 5.10. In this derivation the PLTE relation between the electron temperature and electron density has been used, as well as the values from Hermann's calculations [26]. Also given in this figure is the theoretical relation of $K_{1}^{\text {th }}$ following van der Sijde [41].

In the higher temperature range the experimental points are about a factor 2 below the theoretical values. This is in agreement with the suggestion that for the higher temperature region the four level model underestimates the recombination radiation. In the temperature range which exists in the $40 \mathrm{~A}$ arc and where the diffusion indeed is the main contribution the agreement is better. Although the accuracy in the determination of $K_{1}^{\exp }$ can be improved, e.g. by more detailed knowledge of the escape factor for recombination radiation, we have demonstrated here that $K_{1}$ can be determined from a relatively simple experiment.

\subsection{Results from the Interferometry}

With the interferometric set-up described in 4.3, values of $n_{\mathrm{e}}$ were obtained as a function of the arc current. The accuracy of this determination is better than that of the spectroscopic results and amounts to about $3 \%$. The dominant factor in the accuracy in these measurements is the uncertainty in the arc length. Only when measurements with different values of the arc length are performed, the accuracy can be improved [48]. Our results are based on measurements with two different arc lengths, viz. 58 and $84 \mathrm{~mm}$ for both arc diameters of 5 and $8 \mathrm{~mm}$.

Figure 5.11 shows these interferometric results as a function of the arc current for arc diameters of 5 and $8 \mathrm{~mm}$. Also shown in these figures are the measurements by Nick [38] and our results from the spectroscopic methods. As can be seen the agreement is quite good. Helbig [49] compared the results of interferometric measurements of three laboratories (Kiel, Toulouse and Eindhoven), which are performed with identical or comparable arc sources, with various wavelengths and various types of interferometers, viz. a two wavelength Michelson laser interferometer and a two wavelength coupled-cavity laser interferometer. He concluded that no systematic errors were involved with our used coupledcavity interferometer experiment.

We would like to note here that, since the accuracy in the interferometric results of $n_{\mathrm{e}}$ is better, these results can be used in combination

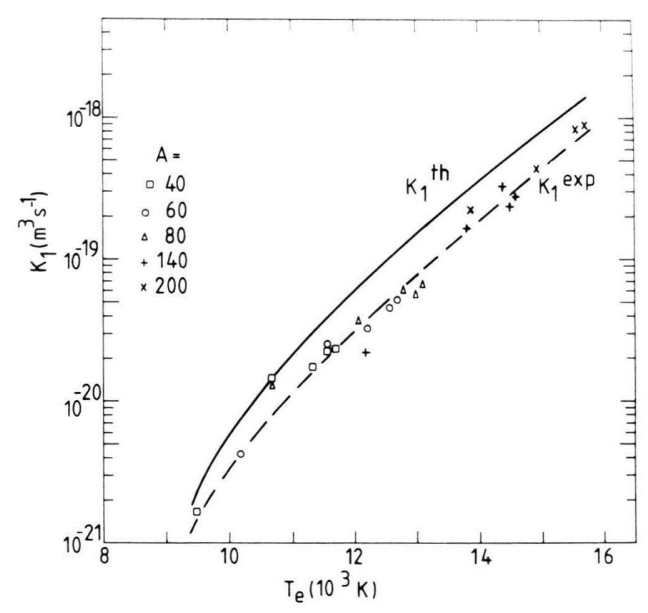

Fig. 5.10. Measured total excitation and direct ionization cross-section for the argon neutral ground state as a function of $T_{\mathrm{e}}$. Also shown is the theoretical relation of $K_{1}^{\text {the }}$ and $T_{\mathrm{e}}[41]$. 


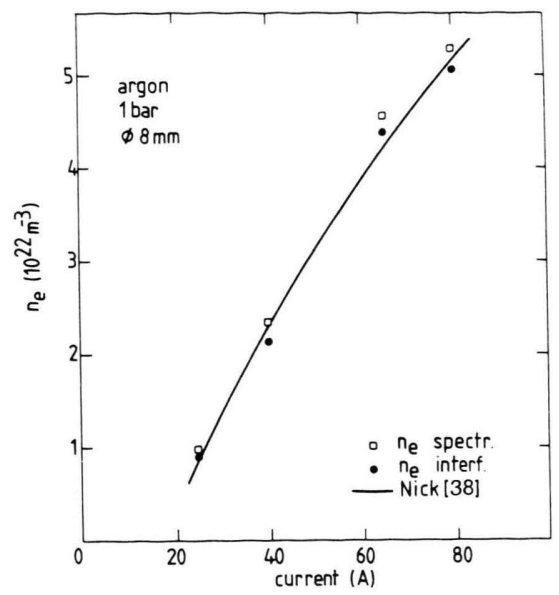

(a)

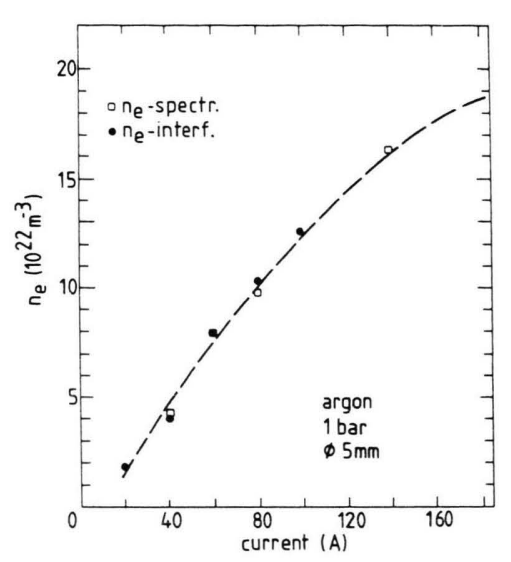

(b)

Fig. 5.11 a, b. Comparison of values of $n_{\mathrm{e}}$ determined by the two wavelength $\mathrm{He}-\mathrm{Ne}$ interferometry and by the spectroscopic methods used in this work and Nick [38] for a 5 and a $8 \mathrm{~mm}$ diameter arc.

with Fig. 5.2 to obtain a precise temperature determination. As has been stated before in 5.2, the $T_{\mathrm{e}}-n_{\mathrm{e}}$-relationship is rather insensitive to small errors in the measurement procedure of the source function method. So, because this interdependence at the relevant pressure is known (Fig. 5.3), the use of $n_{\mathrm{e}}$ in this figure can yield accurate values of $T_{\mathrm{e}}$.

[1] W. A. Cilliers, J. D. Hey, and J. P. S. Rash, J. Quant. Spectrosc. Radiat. Transfer 15, 963 (1975).

[2] H. R. Griem, Plasma Spectroscopy, MacGraw Hill, New York 1964.

[3] M. Venugopalan, Reactions under Plasma Conditions, John Wiley \& Sons, New York 1971.

[4] J. Bacri and M. Lagreca, J. Phys. D. Appl. Phys. 16, 829 (1983).

\section{Conclusions}

1. Deviations from local thermal equilibrium (LTE) can be measured by the source function method, even for systems where these deviations are small.

2. The deviations from LTE for the argon neutral system are mainly caused by recombination radiation to the ground level especially near the arc axis, and by inward diffusion of the neutrals. Radiative recombination is more pronounced at high values of the temperature, while diffusion effects dominate at low values. The observed deviations from LTE of the ground level density are in reasonable agreement with the deviations predicted by a model in which diffusion, radiative recombination and resonance radiation are taken in to account. These three effects are assumed to be the major causes of the non-equilibrium in the argon neutral system.

3. With the source function method $T_{\mathrm{e}}$ and $n_{\mathrm{e}}$ have been determined. The relation between the $T_{\mathrm{e}}$ and $n_{\mathrm{e}}$ appears to be rather independent of the radial position in the arc.

4. An accurate determination of $T_{\mathrm{e}}$ can be obtained by the following procedure:

A measurement of the $n_{\mathrm{e}}$ using an interferometric method. A derivation of $T_{\mathrm{e}}$ from this $n_{\mathrm{e}}$ with their relationship obtained with the source function method.

\section{Acknowledgements}

We are indepted to Dr. V. Helbig (Univ. Kiel, $\mathrm{BRD})$ for his cooperation in the interferometric part of this experiment and for the many valuable discussions involved with this work. We gratefully acknowledge the skillfull technical assistance of J. J. Bleize, L. A. Bisschops and to A. B. M. Hüsken for his indispensable electronic assistance. The authors appreciate the assistance with the calculations done by the students P. M. Vallinga, A. T. M. Wilbers and L. Bol.

[5] P. Fauchais and J. Rakowitz, XIV. ICPIG, Suppl. J. de Phys. C 7, 289 (1979).

[6] H. J. Oskam and E. Pfender, Workshop on Chemistry and Arc Technology, University of Minnesota, Minneapolis, Minnesota, 1980

[7] H. Kaase, Optik 59, 1 (1981).

[8] V. Helbig and K. P. Nick, J. Phys. B: At. Mol. Phys. 14, 3573 (1981). 
[9] J. M. Bridges and W. R. Ott, Applied Optics 16, 367 (1977).

[10] J. M. Bridges, W. R. Ott, E. Pitz, A. Schulz, D. Einfield, and D. Stuck, Applied Optics 16, 1788 (1977).

[11] W. R. Ott, National Bureau of Standards, Washington, D.C. 20234, SP456, 107 (1976).

[12] R. D. Saunders, W. R. Ott, and J. M. Bridges, Applied Optics 17, 593 (1978).

[13] R. Wilson, J. Quant. Spectrosc. Radiat. Transfer 2, 477 (1962).

[14] H. W. Drawin, Z. Physik 225, 470 (1969).

[15] M. Cacciotore, M. Capitelli, and H. W. Drawin, Physica 84 c, 267 (1976).

[16] T. L. Eddy, E. Pfender, and E. R. G. Eckart, IEEE Trans. Plasma Science 1, 31 (1973).

[17] J. F. Uhlenbusch, E. Fischer, and J. Hackmann, Z. Phys. 239, 120 (1970).

[18] J. F. Uhlenbusch, Gaseous Electronics, North-Holland Publishing Company, Amsterdam 1974.

[19] H. W. Drawin, Phys. Letters 42 A, 423 (1973).

[20] J. Leclair and D. C. Schram, XIIIth ICPIG, Berlin, 483 (1977).

[21] R. C. Preston, J. Quant. Spectrosc. Radiat. Transfer 18, 337 (1977).

[22] K.-P. Nick, J. Richter, and V. Helbig, J. Quant. Spectrosc. Radiat. Transfer 32, 1 (1984).

[23] H. W. Drawin and P. Felenbok, Data for Plasmas in Local Thermodynamic Equilibrium, Gauthier-Villars, Paris 1965.

[24] R. J. Rosado, An investigation of non-equilibrium effects in thermal argon plasmas. Ph.D. thesis, Eindhoven Univ. of Technology, Eindhoven, The Netherlands 1981.

[25] C. J. Timmermans, An investigation of pulsed high density plasmas. Ph.D. thesis, Eindhoven Univ. of Technology, Eindhoven, The Netherlands 1984.

[26] W. Hermann, Z. Physik 216, 33 (1968).

[27] I. I. Sobelman, Introduction to the theory of atomic spectra, Pergamon Press, Oxford 1972.

[28] G. A. M. Eilers, J. J. de Jong, R. Kool, and H. N. Linssen, Eindhoven Univ. of Techn. Eindhoven, The Netherlands, Cosor note R 75-22, 1975.

[29] D. W. Marquardt, SIAM J. 11, 431 (1963).

[30] J. C. de Vos, The emissivity of tungsten ribbon, Ph.D. thesis, Univ. of Utrecht, The Netherlands 1953, partly published in Physica 20,690 (1954).
[31] D. Hofsaess, J. Quant. Spectrosc. Radiat. Transfer 19, 339 (1978).

[32] S. E. Schnehage, M. Kock, and E. Schulz-Gulde, J. Phys. B: At. Mol. Phys. 15, 1131 (1982).

[33] M. Abramowitz and I. A. Stegun, Handbook of Mathematical functions, Dover publications Inc., New York 1964.

[34] W. Gautschi, J. Numer. Anal. 7, 187 (1970).

[35] D. E. F. T. Ashy and D. F. Jephcott, Appl. Phys. Lett. 3, 13 (1963).

[36] V. Helbig, Internal report Univ. of Techn., Eindhoven, The Netherlands, Department of Physics, VDF/NT79-23, 1979.

[37] W. Wiese, M. W. Smith, and B. M. Miles, Atomic Transition Probabilities, NSRDS-NBS 22 (1969).

[38] K. P. Nick, Private communication, Diplomarbeit, Christian-Albrechts University, Kiel, BRD (1979).

[39] R. S. Devoto, The Physics of Fluids 9, 1230 (1965).

[40] D. B. Gurevich and I. V. Podomoshenskii, Opt. and Spectrosc. 15, 319 (1963).

[41] B. van der Sijde, J. J. A. M. van der Mullen, and D. C. Schram, Beitr. Plasmaphys. 24, 447 (1984).

[42] K. Katsonis, Etude statistique et cinetique des plasmas d'argon en dehors de l'equilibre thermodynamique local, Ph.D. thesis, Univ. Paris-Sud, Centre D’Orsay, France 1976.

[43] V. M. Batenin and P. V. Minaev, High Temperature 7, 553 (1969).

[44] M. Klein, Radiation trapping processes in the pulsed ion laser, Ph.D. thesis, Univ. of California, USA 1969.

[45] L. Bober and R. S. Tankin, J. Quant. Spectrosc. Radiat. Transfer 10, 991 (1970).

[46] J. F. Uhlenbusch and C. Fischer, Proc. IEEE 59, 578 (1971)

[47] K. P. Nick, Analyse des Plasmazustandes and Bestimmung atomarer Konstanten in einem ArgonKaskadenbogen, Ph.D. thesis, Univ. of Kiel, BRD 1982.

[48] A. Gleizes, Physica 111 C, 386 (1981).

[49] V. Helbig, K. P. Nick, J. Richter, R. J. Rosado, D. C. Schram, and H. Kafrouni, Proc. Vth ESCAMPIG, Dubrovnik, Yugoslavia, 130 (1980). 